\title{
MUC1 is associated with TFF2 methylation in gastric cancer
}

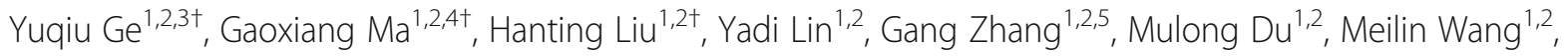
Haiyan Chu ${ }^{1,2^{*}}$, Haiyan Zhang ${ }^{6^{*}}$ and Zhengdong Zhang ${ }^{1,2^{*}}$

\begin{abstract}
Background: Emerging evidence has shown that MUC1 and TFF2 play crucial roles in the H. pylori-infected pathogenesis of gastric cancer (GC). A recent study revealed that H. pylori infection induced obviously increased Tff2 methylation levels in $\mathrm{MuCl}^{-1-}$ mice compared with controls. However, little is known of the molecular mechanism on MUC1 regulating the expression of TFF2.
\end{abstract}

Methods: We conducted a correlation analysis of MUC1 and TFF2 in public databases and our adjacent GC tissues. Besides, MUC1 overexpression vector or small interfering RNA (siRNA) was transfected into GC cells to assess the change in TFF2 expression. Furthermore, the methylation status of TFF2 was measured by bisulfite sequencing PCR (BSP).

Results: The expression of MUC1 was significantly lower in non-cardia and cardia tumor tissues than that in normal tissues. Downregulation of TFF2 expression was also observed in GC tissues. In addition, we found that MUC1 expression was positively associated with TFF2 expression in GC tissues, especially among GC patients with $H$. pylori infection. Overexpression of MUC1 in BGC-823 and SGC-7901 cell lines substantially increased the TFF2 expression, whereas knockdown of MUC1 reverted this effect. Moreover, MUC1 was negatively related to the methylation of TFF2 in the co-expression analysis. The results of BSP experiments showed that compared with negative vector group, the methylation level of TFF2 was decreased in GC cells transfected with MUC1 overexpression vector. Additionally, survival analysis indicated that GC patients with lower level of MUC1 or TFF2 had a worse outcome.

Conclusion: Our results indicated that MUC1 was associated with the methylation of TFF2, which may have implications for TFF2 expression in GC. These findings warrant further research toward the underlying mechanism of MUC1 influenced the TFF2 methylation.

Keywords: MUC1, TFF2, Methylation, Gastric cancer, Molecular epidemiology

\footnotetext{
* Correspondence: chy_grape@njmu.edu.cn; zhyxiaohua@126.com; drzdzhang@njmu.edu.cn

${ }^{\dagger}$ Yuqiu Ge, Gaoxiang Ma and Hanting Liu contributed equally to this work. 'Department of Environmental Genomics, School of Public Health, Jiangsu Key Laboratory of Cancer Biomarkers, Prevention and Treatment, Collaborative Innovation Center For Cancer Personalized Medicine, Nanjing Medical University, 101 Longmian Avenue, Jiangning District, Nanjing 211166, China

${ }^{6}$ Department of Gastroenterology, Xuzhou Clinical College of Nanjing Medical University, Xuzhou Central Hospital, 199 Jiefang South Road, Xuzhou 221009, China

Full list of author information is available at the end of the article
}

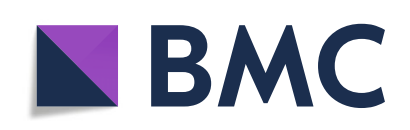

(- The Author(s). 2020 Open Access This article is licensed under a Creative Commons Attribution 4.0 International License, which permits use, sharing, adaptation, distribution and reproduction in any medium or format, as long as you give appropriate credit to the original author(s) and the source, provide a link to the Creative Commons licence, and indicate if changes were made. The images or other third party material in this article are included in the article's Creative Commons licence, unless indicated otherwise in a credit line to the material. If material is not included in the article's Creative Commons licence and your intended use is not permitted by statutory regulation or exceeds the permitted use, you will need to obtain permission directly from the copyright holder. To view a copy of this licence, visit http://creativecommons.org/licenses/by/4.0/. The Creative Commons Public Domain Dedication waiver (http://creativecommons.org/publicdomain/zero/1.0/) applies to the data made available in this article, unless otherwise stated in a credit line to the data. 


\section{Background}

Although the incidence and mortality rates of gastric cancer (GC) are decreased over the past few decades, $\mathrm{GC}$ is still a severe public health problem. Reportedly, GC is the third leading cause of cancer death with the highest incidence occurring in Eastern Asia [1, 2]. GC is a complex disease, arising from the interaction of environmental and genetic factors. It has been established that $H$. pylori infection is the most important risk factor for GC [3]. Accumulating evidence has shown that eradication of $H$. pylori infection was significantly associated with the reduced incidence of GC, highlighting the pathogenic role of $H$. pylori in GC $[4,5]$.

The tumorigenesis progression of GC is involved in multiple stages and develops from normal epithelia, chronic gastritis, atrophic gastritis, intestinal metaplasia, and dysplasia to carcinoma [6, 7]. The original progression of GC is gastric mucosa lesions. Thus, it is necessary to maintain the function of gastric mucosa, which prevents infection by the variety of microorganisms. Mucin glycoproteins are the key components of the mucosal barriers [8]. Mucin 1 (MUC1) is a kind of cell surface mucins and is abundant in mucosal tissues, especially in normal gastric epithelial cells [9]. It has been reported that $M U C 1$ played a crucial role in $H$. pylori-associated gastritis through acting as a protective physical barrier against pathogens $[8$, 10]. Previous study has shown that colonization of $H$. pylori in $M U C 1$-deficient $\left(\mathrm{Mucl}^{-/-}\right)$mice was remarkably higher than that in $\mathrm{Mucl}^{+/+}$mice. Besides, $\mathrm{MUC1}$ was also implicated in decreasing the inflammation induced by H. pylori [11]. In addition, it is noteworthy that previous genome-wide association studies (GWASs) have identified several risk loci located in $M U C 1$ gene, which were significantly associated with the susceptibility of GC $[12,13]$.

Trefoil factor family 2 (TFF2), a member of secreted peptides, is also expressed in gastric mucosa and triggers cell migration signaling to promote epithelial repair [14, 15]. It has been documented that $H$. pylori infection contributes to methylation and silencing of TFF2, thus involving in GC development [16]. Garrett et al. demonstrated that MUC1 acted as a negative regulator of the NLRP3 inflammasome activation and protected against $H$. pylori pathogenesis. In addition, interleukin (IL)-1 promoted precancerous progression in $\mathrm{Mucl}^{-/-}$mice through regulating the methylation status and expression of Tff2 [17]. However, the molecular mechanism of $M U C 1$ involved in the regulation of TFF2 is largely unexplored. Therefore, we hypothesized that the aberrant MUC1 regulates the methylation status of TFF2, thus contributes to silence of TFF2 in the carcinogenesis of GC. In the present study, we demonstrated that both $M U C 1$ and TFF2 were downregulated in GC tissues. Overexpression of $M U C 1$ was significantly associated with decreased the TFF2 methylation and increased the TFF2 expression.

\section{Materials and methods}

\section{Samples and datasets}

This study was approved by the international review board of Nanjing Medical University. A total of 59 GC patients were recruited from the Second Affiliated Hospital of Nanjing Medical University. We detected the expression of MUC1 and TFF2 in adjacent GC tissues and compared the difference of expression between $H$. pylori infected $(n=31)$ and non-infected $(n=28)$ GC patients. The RNAseq data from The Cancer Genome Atlas (TCGA) database was acquired by the UCSC Xena project (http://xena. ucsc.edu/). In this study, we downloaded gene expression $(n=417$, IlluminaHiSeq RNASeq platform) and DNA methylation $(n=398$, Illumina Infinium HumanMethylation450 platform) profiles. Samples with both gene and methylation expression profiles were included in the further correlation analysis $(n=373)$. Besides, we analyzed gene expression array deposited in Gene Expression Omnibus (GEO, GSE29272), which has the global gene expression of 72 gastric non-cardia tumor and 62 gastric cardia tumor and their paired normal tissues [18]. We also applied a GEO dataset (GSE74577), which was conducted to investigate the gene expression changes in response to H. pylori infection [19]. Additionally, GSE99553 was used to compare methylation level of $\mathrm{CpG}$ sites between GC patients with $H$. pylori infection $(n=28)$ and non-infected patients $(n=28)$.

\section{Cell culture and transfection}

The full-length MUC1 cDNA was synthesized and constructed into the pEGFP-N1 vector. GC cell lines (BGC823 and SGC-7901) were transfected with overexpression vector using Lipofectamine 2000 reagent (Invitrogen, Carlsbad, CA, USA). Vectors in this study were confirmed by DNA sequencing. Small interfering RNA (siRNA) was synthesized to inhibit MUC1 expression. The sequence of siRNA is the following: CGGGAT ACCTACCATCCTA.

\section{Quantitative real-time PCR (qRT-PCR) analysis}

Total RNA from BGC-823 and SGC-7901 cell lines was isolated by Trizol Reagent (Invitrogen, CA, USA), and cDNA was synthesized by Primescript RT Reagent Kit (TaKaRa, Osaka, Japan). The real-time PCR was conducted with ABI 7900HT Real-Time PCR System (Applied Biosystem, Foster City, CA, USA) using SYBR Green assays. GAPDH was treated as internal control to quantify the expression levels of TFF 2 and MUC1 by $2^{-\Delta c t}$ method. The primer sequences are as follows: F: 5'-GCTGTT TCGACTCCAGTGTCA-3' and R: 5'-CCACAGTTTCT TCGGTCTGAG-3' for TFF2, F: 5'-CCTACCATCCTAT GAGCGAGTAC-3' and R: 5' -GCTGGGTTTGTGTAAG AGAGGC-3' for $M U C 1$, F: 5'-CCGGGAAACTGTGG 
CGTGATGG-3' and R: 5'-AGGTGGAGGAGTGGGTG TCGCTGTT-3' for GAPDH.

\section{Bisulfite sequencing PCR (BSP)}

DNA was isolated from BGC-823 and SGC-7901 cell lines by DNeasy Blood \& Tissue Kit (QIAGEN, Hilden, Germany) according to the manufacturer's protocols. We used EpiTect Fast DNA Bisulfite Kit (QIAGEN) to accomplish bisulfite conversion of genomic DNA. The primers for amplification are shown in Supplementary Table 1. Then, the PCR products were purified and cloned into pTG19-T Vector (Generay, Co., Ltd., Shanghai, China). Ten subclones from each cell line with two replicated experiments were chosen for sequence. Methylation level was calculated as the number of
CPG methylated loci divided by the number of detected CpG loci.

\section{Statistical analysis}

Student's $t$ test or paired $t$ test was used to compare the differences of gene expression or methylation level. Associations of gene expression and methylation level of CpG sites were assessed by Pearson's correlation analysis. We applied an online tool, Kaplan-Meier Plotter, to calculate hazard ratios (HRs) and their correspondence $95 \%$ confidence interval (95\% CI) [20]. The logrank test was applied to assess the significant effect of gene expression level on survival of patients. We used multivariate cox regression analysis to assess the prognostic value of MUC1 and TFF2, with adjustment for

\section{A}

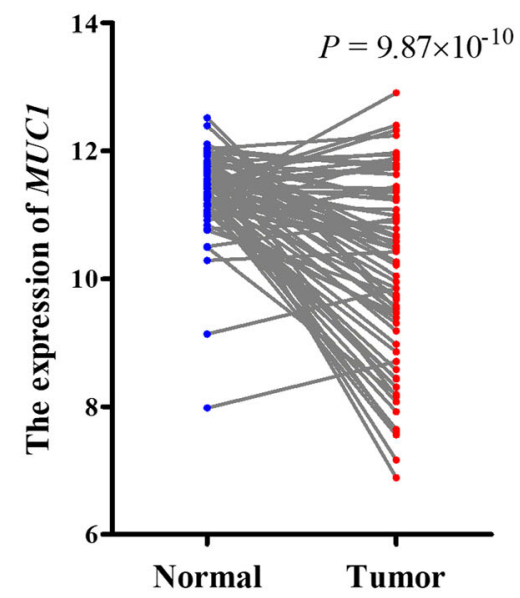

C

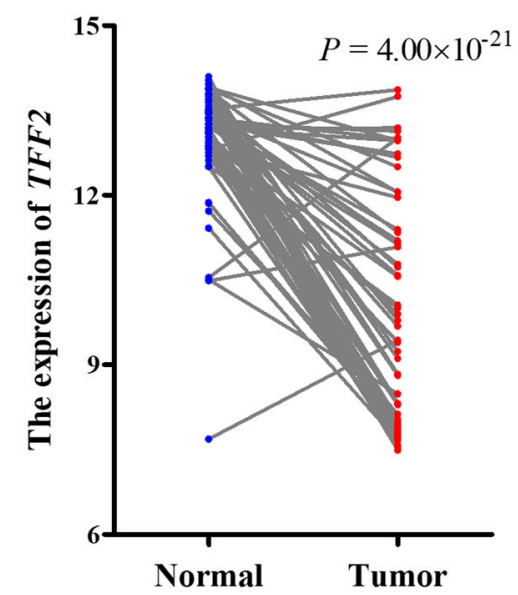

B

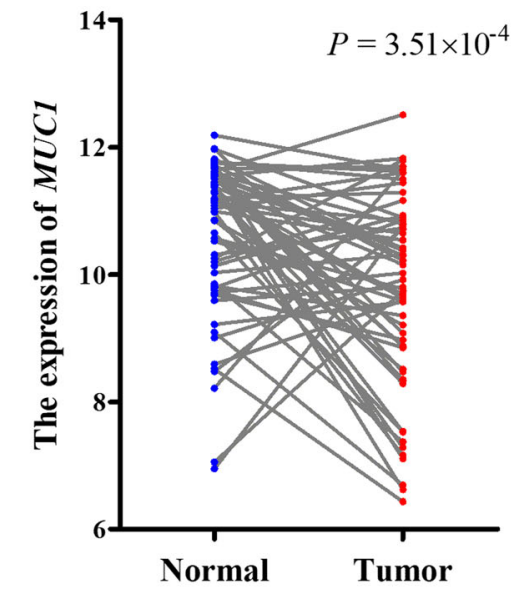

D cardia

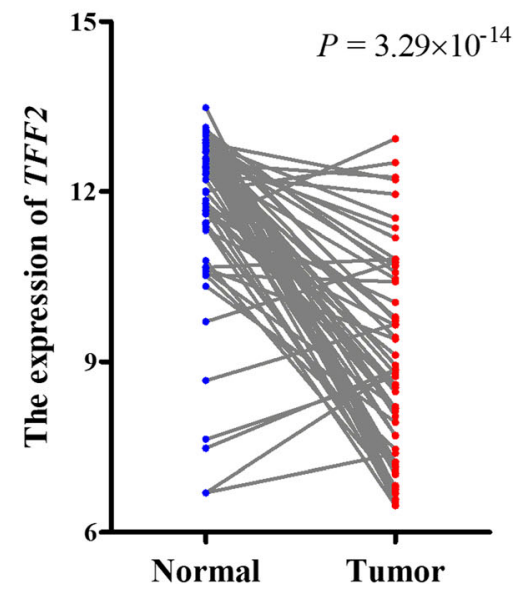

Fig. 1 Downregulation of MUC1 and TFF2 in GC tissues. The expression level of MUC1 was significantly lower in non-cardia (a) and cardia (b) tumor tissues than that in adjacent normal tissues. $\mathbf{c}$, d Downregulation of TFF2 expression in non-cardia (c) and cardia (d) tumor tissues. All $P$ values were from paired $t$ tests 
age, sex, grade, and stage of GC patients. All statistical analyses were conducted by the $\mathrm{R} 3.5 .1$ software, and two-tailed $P$ value $<0.05$ was considered as statistically significant.

\section{Results}

\section{Downregulation of $\mathrm{MUC1}$ and TFF2 in GC}

We assessed the expression level of MUC1 and TFF2 in gastric non-cardia and cardia tissues and their correspondence normal tissues from GEO database (GSE29272). As shown in Fig. 1, the expression of $M U C 1$ in non-cardia tumor tissue was significantly lower than that in paired adjacent normal tissues $\left(P=9.87 \times 10^{-10}\right.$; Fig. 1a). Additionally, compared with normal tissues, a similar downregulated expression of $M U C 1$ was observed in the cardia tumor tissue $\left(P=3.51 \times 10^{-4}\right.$; Fig. $\left.1 \mathrm{~b}\right)$. As with $M U C 1$, TFF2 was significantly decreased in non-cardia and cardia tumor tissues compared with that in adjacent normal tissues $\left(P=4.00 \times 10^{-21}\right.$ and $P=3.29 \times 10^{-14}$, respectively; Fig. 1c, d). Both $M U C 1$ and TFF2 expression showed a slight decreased in cardia tissue compared with noncardia tumor tissue, but there were no significantly differences (Supplementary Figure 1).

Additionally, we detected the expression of $M U C 1$ and TFF2 in adjacent GC tissues from our cohort. Compared with non-infected patient, MUC1 and TFF2 expression were not statistically significant and showed a slightly decreased trend in H. pylori-infected patients (Supplementary Figure 2A-B). The results of GEO dataset (GSE74577) indicated that the expression of $M U C 1$ but not TFF2 was significantly decreased in GES-1 cells after 24-h infection with $H$. pylori $\left(P=4.49 \times 10^{-4}, P=0.582\right.$, respectively; Supplementary Figure 2C-D). Besides, we found the methylation level of CpG sites (cg13804478, cg18804777, and cg24512973) located in MUC1 were significantly higher in $H$. pylori-infected patients than in non-infected patient (Supplementary Figure 3). For TFF2, there was one CpG site; cg26403416 was significantly increased in $H$. pylori-infected patients than in non-infected patients in both GSE99553 and TCGA datasets (data was not shown).

\section{MUC1 positively regulates the expression of TFF2}

In the present study, we conducted the co-expression between MUC1 and TFF2 in the GSE29272 database. The correlation analysis results showed that $M U C 1$ expression was positively associated with TFF2 expression in GC tissues $\left(r=0.425, P=3.20 \times 10^{-7}\right.$; Fig. $\left.2 \mathrm{a}\right)$. The similar positive relationship between $M U C 1$ and TFF2 was found in the TCGA database $\left(r=0.449, P=3.13 \times 10^{-20}\right.$; Fig. $\left.2 b\right)$. In addition, we investigate the relationship between the expression of MUC1 and TFF2 in our cohort. The result

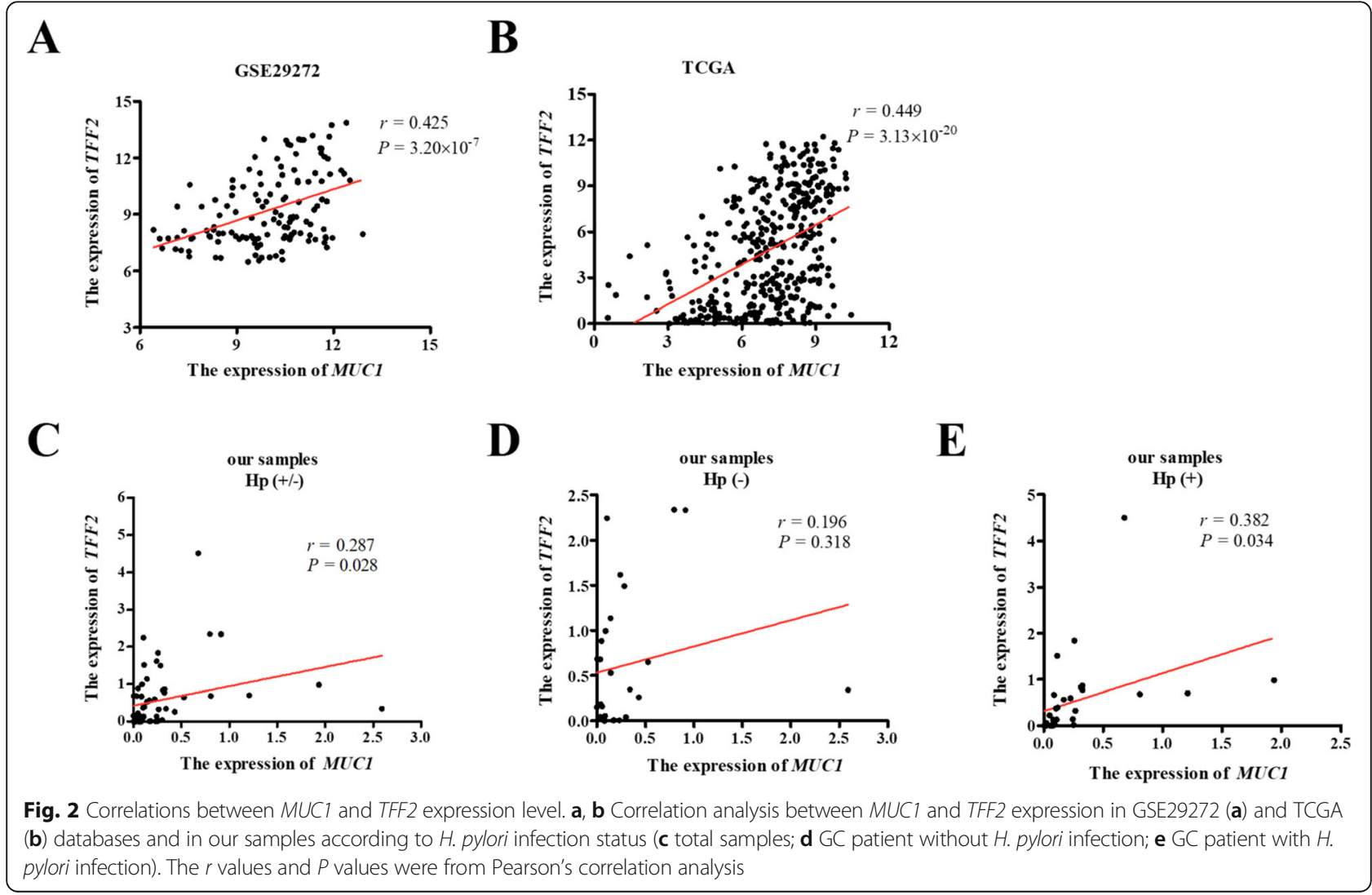


demonstrated significantly positive correlation of $M U C 1$ and TFF2 expression among GC patients with $H$. pylori infection but not for those without infection (total: $r=$ $0.287, P=0.028$; $H$. pylori negative: $r=0.196, P=0.318 ; H$. pylori positive: $r=0.382, P=0.034$; Fig. $2 \mathrm{c}-\mathrm{e})$. Furthermore, the positive correlations between the expression of $M U C 1$ and TFF2 were also observed in other clinical features, including different age, sex, stage, and grade of GC patients (Supplementary Table 2).

We next assessed whether $M U C 1$ positively regulated the TFF2 expression in two GC cell lines (BGC-823 and SGC-7901). The TFF2 expression level was measured by qRT-PCR after GC cell lines transfected with $M U C 1$ overexpression vector or siRNA. The results showed that overexpression of MUC1 in BGC-823 and SGC-7901 cell lines substantially increased the TFF 2 expression, whereas inhibition of $M U C 1$ reverted this effect (Fig. 3).

\section{Overexpression of MUC1 promotes hypomethylation of TFF2}

We investigated whether the methylation levels of $M U C 1$ was associated with the dysregulation of $M U C 1$. The results indicated that seven CpG sites were negatively associated with the MUC1 expression (Supplementary Table 3). Given that high $M U C 1$ expression is correlated with increased the TFF2 level, we then evaluated the

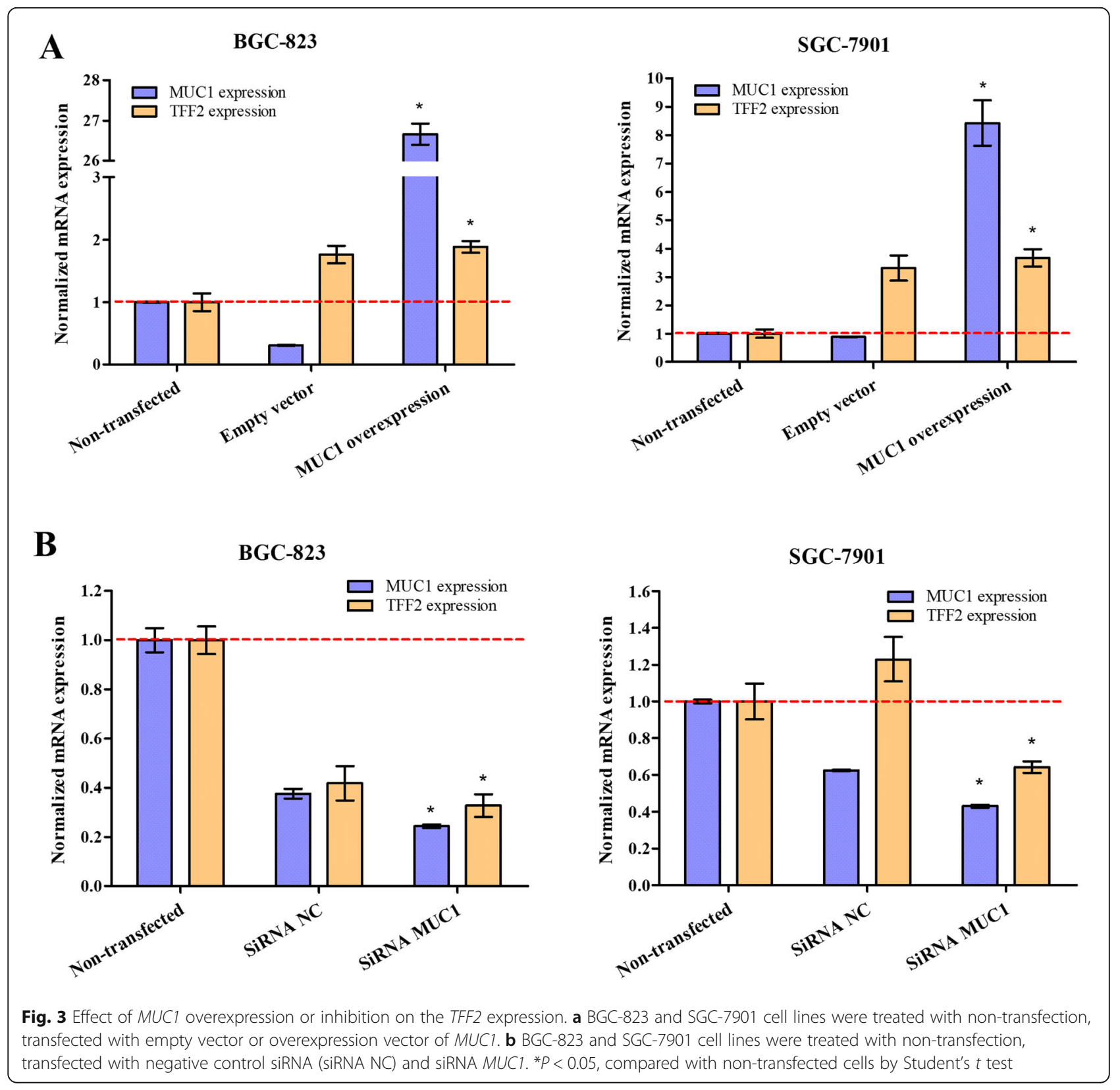


associations of methylation level of TFF2 and MUC1 expression. The detail description of $\mathrm{CpG}$ sites located in TFF2 gene is summarized in Supplementary Figure 4. As presented in Table 1 and Fig. 4, significant associations between MUC1 expression and methylation levels of cg14721139, cg11158374, cg12456510, cg26403416, cg18879389, and cg09410308 were identified in GC tumor sample (all $P<0.05)$. Furthermore, we found that the expression level of TFF2 was negatively related to the methylation status of these six CpG sites (all $P<0.05$; Supplementary Figure 5). Subsequently, we conducted the correlation analysis of these six CpG sites themselves, and the results showed that the methylation level of these six CpG between each other were all prominently positive correlated (Supplementary Figure 6).

To better understand of the regulatory mechanism, BSP was performed to compare the methylation level in GC cell lines transfected with negative vector or MUC1 overexpression vector. In line with the correlation analysis, BSP results revealed that overexpression of $M U C 1$ contributed to a remarkable decrease in methylation of CpG site located in TFF2 in BGC-823 cells $(P=0.041)$, and similar reduce trend was observed in SCG-7901 cell line $(P=0.078)$ (Table 2).

We also detected the effect of $M U C 1$ on expression of DNA methylation transferases (DNMTs), including DNMT1, DNMT3a, and DNMT3b. The results indicated that both overexpression and inhibition of $M U C 1$ leaded to a slight increase trend of DNMTs, especial DNMT3a (Supplementary Figure 7). These results suggested that MUC1 was not associated with DNMTs expression.

\section{Downregulation of MUC1 and TFF2 are associated with poor outcome of GC}

We further investigated the prognosis effect of $M U C 1$ and TFF2 on survival of GC patients by utilizing an online tool, Kaplan-Meier Plotter, which integrated gene

Table 1 Correlation analysis of methylation levels of TFF2 with MUC1 expression in gastric tumor tissues

\begin{tabular}{lllll}
\hline CpG site & Position & Gene region & Coefficient & $P^{\mathrm{a}}$ \\
\hline cg14721139 & Chr21:43772498 & Promoter & -0.270 & $1.18 \mathrm{E}-07$ \\
cg12234272 & Chr21:43772018 & Promoter & 0.026 & 0.622 \\
cg00355325 & Chr21:43771923 & Promoter & 0.032 & 0.540 \\
cg11158374 & Chr21:43771664 & Promoter & -0.170 & $9.72 \mathrm{E}-04$ \\
cg12456510 & Chr21:43771356 & Promoter & -0.120 & $1.99 \mathrm{E}-02$ \\
cg26403416 & Chr21:43771340 & Promoter & -0.220 & $1.73 \mathrm{E}-05$ \\
cg18879389 & Chr21:43771121 & Promoter & -0.185 & $3.31 \mathrm{E}-04$ \\
cg00083685 & Chr21:43767586 & Gene body & -0.084 & 0.106 \\
cg09410308 & Chr21:43766606 & Gene body & -0.177 & $5.73 \mathrm{E}-04$ \\
\hline
\end{tabular}

${ }^{a} P$ value for Pearson's correlation analysis expression data and survival information of GC patients. Prominent associations of MUC1 and TFF2 with outcomes of GC patients were obtained. The Kaplan-Meier plot showed that GC patients with high expression of MUC1 had better outcome, with HR of 0.82 (95\% CI, 0.69-0.97) comparing those with low level of $M U C 1$ (logrank $P=0.018$; Fig. 5a). In addition, higher TFF2 expression was also obviously related to lengthen overall survival of GC patients ( $\mathrm{HR}=0.79, \log$-rank $P=0.009$; Fig. 5b).

Furthermore, we divided GC patients into four groups according to the median of $M U C 1$ and TFF2 expression by using TCGA database, including MUC1 low+TFF2 low, MUC1 low + TFF2 high, MUC1 high+TFF2 low, and $M U C 1$ high+TFF2 high. Kaplan-Meier curve showed no significant difference of overall survival between four groups (Supplementary Figure 8). The result of multivariate cox regression analysis is summarized in Supplementary Table 4. After adjustment for age, sex, grade, and TNM stage, the MUC1 and TFF2 expression were not related to survival of GC patients.

\section{Discussion}

There has been wide consensus that both mucins and TFF peptides play crucial roles in protecting mucosal epithelial cells from a variety of insults [21, 22]. In the present study, we demonstrated that the expression of MUC1 and TFF2 were significantly lower in GC tissues than that in adjacent normal tissues. $M U C 1$ positively regulated the expression of TFF2 through correlation and experimental analysis. In addition, we observed that the methylation status of TFF2 was prominent related to the MUC1 expression. Furthermore, consistent with coexpression analysis findings, negative regulation of MUC1 on methylation level of TFF2 was also revealed in our BSP experiment, suggesting the role of $M U C 1$ in mediating TFF 2 expression by decreasing the methylation level of TFF2.

Epidemiology studies have demonstrated that about $50 \%$ of human infects $H$. pylori over the world and severe gastric disease occurs in 10-15\% of infected carriers $[23,24]$. Host factors play a key role in determining the susceptibility of $H$. pylori-infected carriers developed into GC. Mounting evidence shows that cell surface mucins protect gastric epithelial cell by their defense against H. pylori infection [25]. There are multiple members of mucins expressed in a variety of mucosal tissues. Among them, MUC1 is the most widely investigated mucin and is identified to be abundantly expressed in all mucosal tissues [25]. Many studies have reported that the extracellular domain of $M U C 1$ is hypervariable and contains a variety of tandemly repeated 20-amino acid unites, which is well known to be associated with the increased risk of GC [26-28]. Previous GWASs have suggested significant associations between the $M U C 1$ risk loci and 


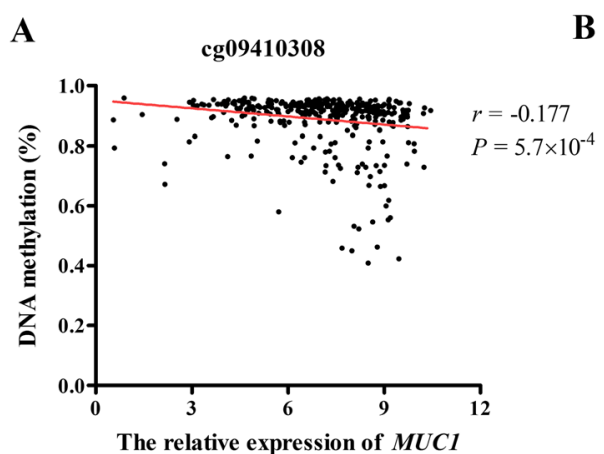

B

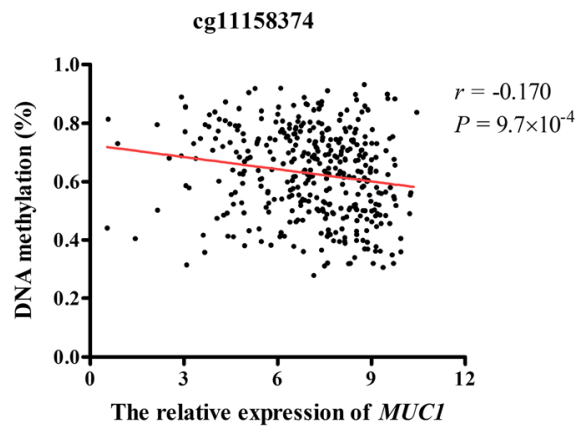

C

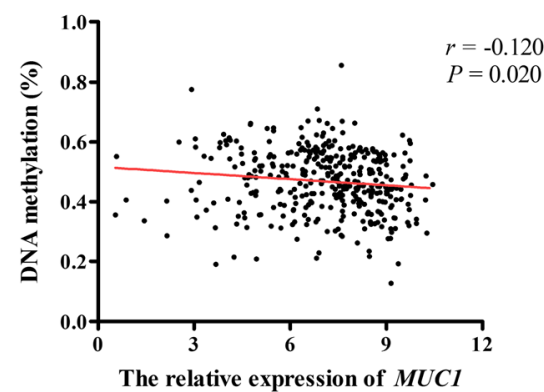

E

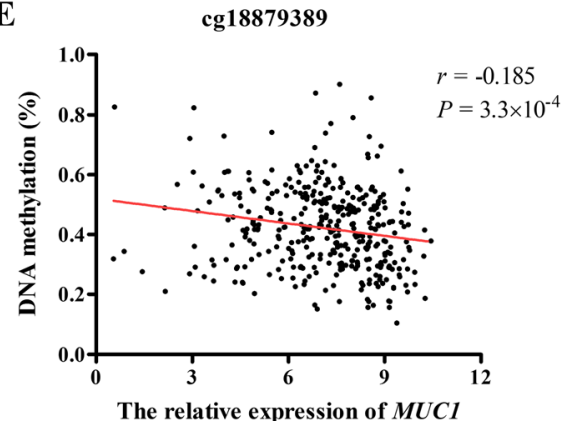

D

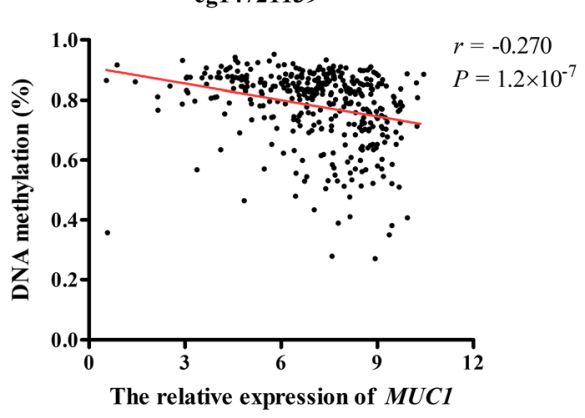

F

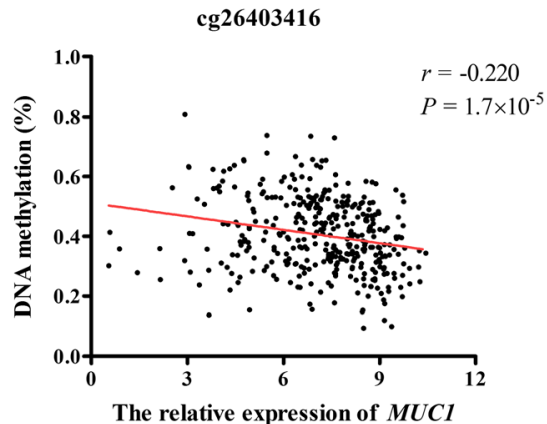

Fig. 4 Correlations between MUC1 expression and methylation level of CpG loci located in TFF2

susceptibility of GC [12, 13]. SNP rs4072037 was reported to be pathogenic in $\mathrm{GC}$ for it altered the alternative splicing and transcriptional regulation of MUC1 [29, 30]. Indeed, our results confirmed that MUC1 was significantly lower in whether cardia or non-cardia tumor tissues than that in adjacent normal tissues. All findings described above highlight the key role of $M U C 1$ in the carcinogenesis of GC.
Epigenetic aberrations also play a key role in the pathogenesis of GC. It is generally agreed upon that $H$. pylori infection induced extensive DNA methylation alterations in gastric epithelial cells [31]. Numerous studies have shown that dysregulated promoter methylation contributed to silencing of tumor suppressor genes [32, 33]. A recent study revealed that $H$. pylori infection increased methylation level and decreased expression of

Table 2 Difference of TFF2 methylation level between control and MUC1 overexpression group

\begin{tabular}{llllllll}
\hline Variables & cg14721139(\%) & cg11158374 (\%) & cg12456510 (\%) & cg26403416 (\%) & cg18879389 (\%) & cg09410308 (\%) & $P^{a}$ \\
\hline BGC-NC & 70 & 55 & 25 & 20 & 10 & 95 & 65 \\
BGC-OE & 25 & 55 & 0 & 10 & 5 & 95 \\
SGC-NC & 70 & 90 & 15 & 10 & 25 & 95 \\
SGC-OE & 40 & 80 & 15 & 5 & 0 & 95 & 0.078 \\
\hline
\end{tabular}

${ }^{\mathrm{a}} P$ value for paired $t$ test 


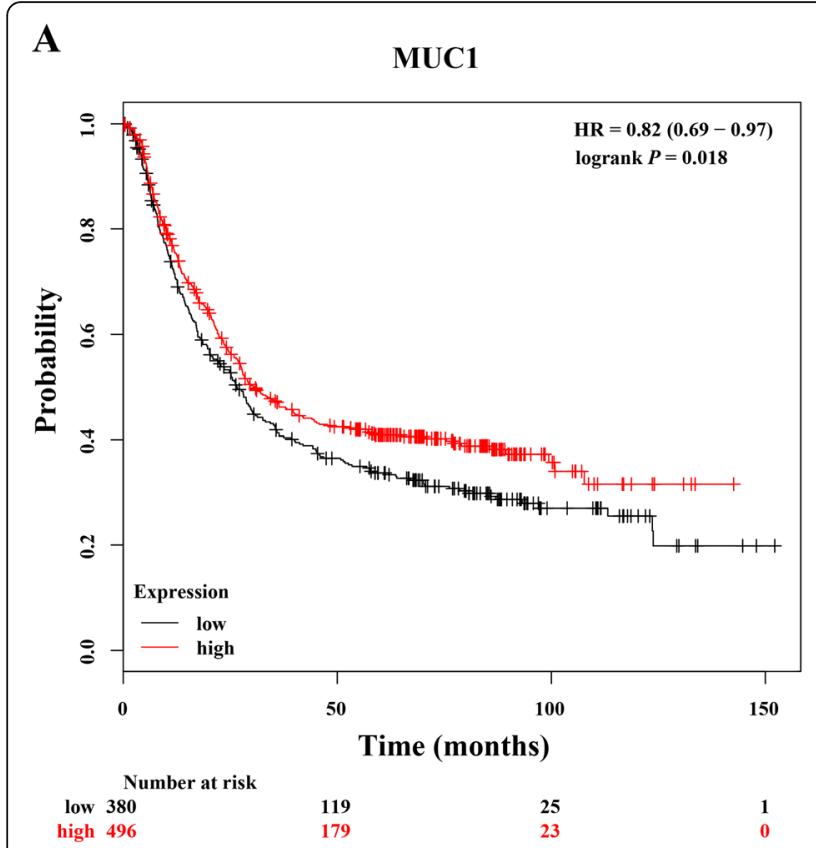

B

TFF2

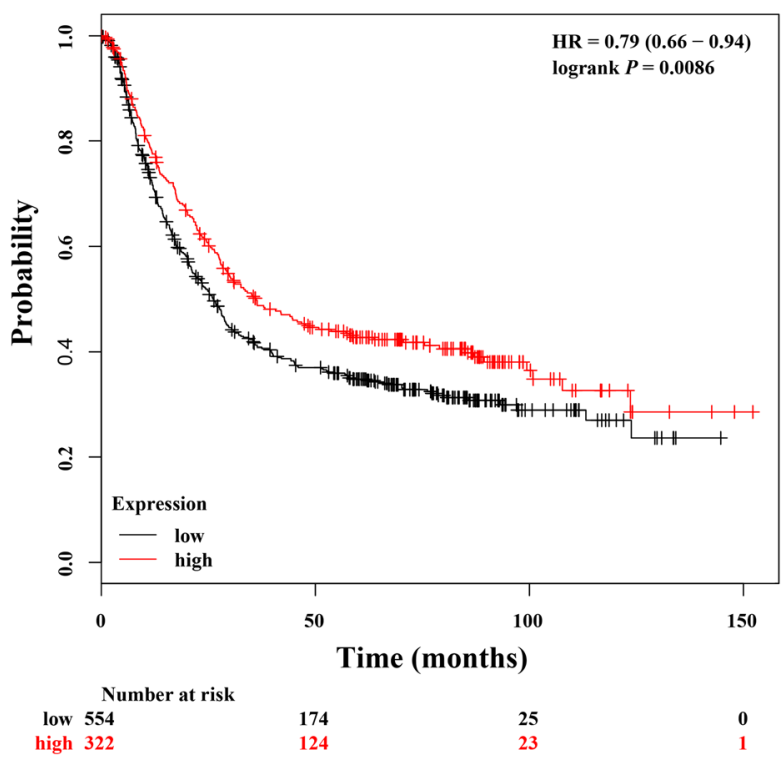

Fig. 5 Kaplan-Meier curve of overall survival in GC patients according to MUC1 and TFF2 expression by Kaplan Meier plotter

TFF2, thus implicating in the development of GC [16]. TFF is a member of secreted protein family, which is composed by TFF1, TFF 2 , and TFF3, and characterized by the trefoil domain [34]. A lot of published studies revealed that the TFFs are involved in multiple biological processes, including barrier protection, cell proliferation, and apoptosis [35-37]. TFF1 is a well-known gastricrelated tumor suppressor gene, whereas the function of TFF2 in GC is relatively less investigated. Accumulating data has shown that TFF2 expression is frequently silenced in GC. In this study, we also found an obvious lower expression level of TFF2 in GC tissues than that in adjacent normal tissue. Besides, previous experiments indicated that $T f f 2^{-1-}$ mice promoted the progression of gastritis to dysplasia after $H$. pylori infection [38]. Another study revealed that TFF2 reduced proliferation of pancreatic ductal adenocarcinoma [39]. Additionally, the tumor suppressor effect of TFF2 was also found in prostate and breast cancer [40, 41].

There has been wide consensus that epithelial cells synthesize and secrete TFF peptides together with mucins [42]. Intriguingly, $\mathrm{Mucl}^{-/-}$mice infected with $\mathrm{H}$. pylori obtained the similar phenotypes that appeared in $\mathrm{Tff}^{-1-}$ mice. Furthermore, comparing with the infected controls, the infected $\mathrm{Mucl}^{-1-}$ mice prominently increased methylation level of TFF2 [17]. Nevertheless, limited study is available to reveal the regulation mechanism between $M U C 1$ and TFF2. Our results showed that significantly positive associations between MUC1 and TFF2 were observed in tumor tissues and GC cell lines. Yamaguchi et al. found that TFF2 was downregulated by promoting methylation of its promoter in pancreatic ductal adenocarcinoma, which was also identified in $H$. pylori-related GC [16, 39]. Considering that aberrant methylation of TFF2 were involved in the carcinogenesis of tumor, we carried out co-expression analysis between $M U C 1$ and methylation of CpG loci located in TFF2. The results showed that the expression level of $M U C 1$ was reversely related to the methylation status of TFF2. It has been established that epigenetic dysregulation in promoter of genes plays an important role in the development of GC [43, 44]. Intriguingly, majority of these significant $\mathrm{CpG}$ loci are located in the promoter region of TFF2. Bioinformatic analysis indicated that transcription factors MAX, TCF12, and EZH2 may bind with the promoter of TFF2 (https://www. genecards.org/). Consistent with findings in the earlier study, negative associations between TFF2 methylation and the expression of TFF 2 were also detected in our correlation analysis. Moreover, BSP experiments further confirmed that overexpression of $M U C 1$ decreased the TFF2 methylation. These results suggested the negatively regulate effect of $M U C 1$ on methylation level of TFF2, contributing to decrease TFF2 levels. Given the important role of MUC1 and TFF2 in the development of GC, we have also investigated their prognosis effect. Our results indicated that GC patients with low level of MUC1 or TFF2 had poorer outcome, indicating that MUC1 and TFF2 may act as prognostic biomarkers in GC.

Some limitations are worthy of attention in the present study. Firstly, because numerous studies have supported the role of mucins and TFF peptide in the H. pylori-related pathologies, we investigated the regulation 
mechanism of $M U C 1$ implicated in the expression of TFF2 without consideration of the $H$. pylori infection. In addition, we assessed co-expression between the MUC1 and TFF2 in public databases and our samples. Although the $P$ value for correlation analysis was statistically significant, the coefficient $(r)$ between MUC1 and TFF2 expression was relatively small. Moreover, this study found that MUC1 could regulate the DNMTs expression slightly, suggesting $M U C 1$ was not associated with the expression of DNMTs. The underlying mechanism of MUC1 influenced the methylation status of TFF2 need to be further explored. Besides, whether $M U C 1$ and TFF 2 could act as the independent prognostic factors for GC remains to be investigated. The clinical studies of multicenter and larger samples are needed to validate our findings.

In conclusion, by using integrative analysis of the expression profiles and molecular experiments, we revealed that $M U C 1$ was negatively associated with the methylation of TFF 2 and positively regulated TFF2 expression in GC. These findings provided new light on the strong link between MUC1 and TFF2 regulation. Further studies are warrant to explore the underlying epigenetic mechanism of this relationship in the pathogenesis of GC.

\section{Supplementary information}

Supplementary information accompanies this paper at https://doi.org/10. 1186/s13148-020-00832-6.

\section{Additional file 1: Table S1. The primers for amplification in BSP}

experiments.

Additional file 2: Table S2. Subgroup analysis of the correlation of MUC1 and TFF2 expression in TCGA database.

Additional file 3: Table S3. Correlation analysis of MUC1 methylation levels with MUC1 expression in GC tissues.

Additional file 4: Table S4. Multivariate cox regression analysis for survival of GC patients in TCGA database.

Additional file 5: Figure S1. The expression of MUC1 (A) and TFF2 (B) in non-cardia and cardia tissue from GSE29272.

Additional file 6: Figure S2. H. pylori infection and the expression of MUC1 and TFF2. (A-B) The MUC1 and TFF2 expression in adjacent GC tissues with or without $H$. pylori infection from our cohort. (C-D) The expression change of MUC1 and TFF2 in GES-1 cells after 24 h-infection with H. pylori (GSE74577).

Additional file 7: Figure S3. The difference of methylation levels of $\mathrm{CpG}$ sites located in MUC1 in GC patients with or without $H$. pylori infection (GSE99553 and TCGA). ${ }^{*} P<0.05$; ${ }^{* *} P<0.01 ;{ }^{* * *} P<0.001$.

Additional file 8: Figure S4. A schematic representation of the $9 \mathrm{CpG}$ sites located in TFF2 gene.

Additional file 9: Figure S5. Correlations between TFF2 expression and methylation level of CpG loci located in TFF2.

Additional file 10: Figure S6. The Correlation analysis of the significant CpG sites located in TFF2. The values showed the Pearson's correlation coefficient and corresponding 95\% confidence interval.

Additional file 11: Figure S7. The normalized mRNA expression of DNMT1, DNMT3a and DNMT36 in BGC-823 and SGC-7901 cell line with MUC1 overexpression (A) or inhibition (B).
Additional file 12: Figure S8. Kaplan Meier curve for overall survival of GC patients in four groups: MUC1 low+TFF2 low; MUC1 low+TFF2 high; MUC1 high+TFF2 low; MUC1 high+TFF2 high from TCGA database.

\section{Abbreviations}

95\% Cl: 95\% confidence interval; BSP: Bisulfite sequencing PCR; DNMTs: DNA methylation transferases; GC: Gastric cancer; GEO: Gene Expression Omnibus; GWASs: Genome-wide association studies; HR: Hazard ratio; MUC1: Mucin 1; NC: Negative control; qRT-PCR: Quantitative real-time PCR; siRNA: Small interfering RNA; TCGA: The Cancer Genome Atlas; TFF2: Trefoil factor family 2

\section{Acknowledgements}

Not applicable.

\section{Authors' contributions}

YG, GM, and HL carried out the most statistical analysis, functional experiments, and drafted the manuscript. YL and HL participated in the extracted RNA and BSP experiment. MD and GZ collected the data and conducted the COexpression analysis. MW contributed in revising the manuscript. $\mathrm{ZZ}, \mathrm{HZ}$, and $\mathrm{HC}$ participated in design, coordination, and review of the manuscript. All authors read and approved the final manuscript.

\section{Funding}

This study was partly supported by the National Natural Science Foundation of China (81230068, 81773539, 81773538, 81903390), the National Key R\&D Program of China (grants 2018YFC1313100, 2018YFC1313102), Collaborative Innovation Center for Cancer Personalized Medicine, and the Project Funded by the Priority Academic Program Development of Jiangsu Higher Education Institutions (Public Health and Preventive Medicine).

\section{Availability of data and materials}

The Cancer Genome Atlas (TCGA) database was available by the UCSC Xena project (http://xena.ucsc.edu/). The Gene Expression Omnibus (GEO) was publicly available online at https:/www.ncbi.nlm.nih.gov/geo/, and the accession numbers are GSE29272, GSE74577, and GSE99553.

Ethics approval and consent to participate

This study was approved by the international review board of Nanjing Medical University.

\section{Consent for publication}

Not applicable.

\section{Competing interests}

The authors declare that they have no competing interests.

\section{Author details}

${ }^{1}$ Department of Environmental Genomics, School of Public Health, Jiangsu Key Laboratory of Cancer Biomarkers, Prevention and Treatment, Collaborative Innovation Center For Cancer Personalized Medicine, Nanjing Medical University, 101 Longmian Avenue, Jiangning District, Nanjing 211166, China. ${ }^{2}$ Department of Genetic Toxicology, The Key Laboratory of Modern Toxicology of Ministry of Education, School of Public Health, Nanjing Medical University, Nanjing, China. ${ }^{3}$ Department of Public Health and Preventive Medicine, Wuxi School of Medicine, Jiangnan University, Wuxi, China. ${ }^{4}$ State Key Laboratory of Natural Medicines, School of Traditional Chinese Pharmacy, China Pharmaceutical University, Nanjing, China. ${ }^{5}$ Department of Neurology, Children's Hospital of Nanjing Medical University, Nanjing, China. ${ }^{6}$ Department of Gastroenterology, Xuzhou Clinical College of Nanjing Medical University, Xuzhou Central Hospital, 199 Jiefang South Road, Xuzhou 221009, China.

Received: 19 August 2019 Accepted: 23 February 2020

Published online: 02 March 2020

\section{References}

1. Torre LA, Bray F, Siegel RL, Ferlay J, Lortet-Tieulent J, Jemal A. Global cancer statistics, 2012. CA Cancer J Clin. 2015;65:87-108. 
2. Ferlay J, Soerjomataram I, Dikshit R, Eser S, Mathers C, Rebelo M, Parkin DM, et al. Cancer incidence and mortality worldwide: sources, methods and major patterns in GLOBOCAN 2012. Int J Cancer. 2015;136:E359-86.

3. Van Cutsem E, Sagaert X, Topal B, Haustermans K, Prenen H. Gastric cancer. Lancet. 2016:388:2654-64.

4. Lee YC, Chiang TH, Chou CK, Tu YK, Liao WC, Wu MS, Graham DY. Association between helicobacter pylori eradication and gastric cance incidence: a systematic review and meta-analysis. Gastroenterology. 2016; 150:1113-24 e1115.

5. Graham DY. Helicobacter pylori update: gastric cancer, reliable therapy, and possible benefits. Gastroenterology. 2015;148:719-31 e713.

6. Correa P. Gastric cancer: overview. Gastroenterol Clin N Am. 2013;42:211-7.

7. Correa P. A human model of gastric carcinogenesis. Cancer Res. 1988;48: 3554-60.

8. McGuckin MA, Linden SK, Sutton P, Florin TH. Mucin dynamics and enteric pathogens. Nat Rev Microbiol. 2011;9:265-78.

9. Gendler SJ. MUC1, the renaissance molecule. J Mammary Gland Biol Neoplasia. 2001;6:339-53.

10. Linden SK, Sheng YH, Every AL, Miles KM, Skoog EC, Florin TH, Sutton P, et al. MUC1 limits helicobacter pylori infection both by steric hindrance and by acting as a releasable decoy. PLoS Pathog. 2009;5:e1000617.

11. McGuckin MA, Every AL, Skene CD, Linden SK, Chionh YT, Swierczak A, McAuley J, et al. Muc1 mucin limits both helicobacter pylori colonization of the murine gastric mucosa and associated gastritis. Gastroenterology. 2007; 133:1210-8.

12. Hu N, Wang Z, Song X, Wei L, Kim BS, Freedman ND, Baek J, et al. Genomewide association study of gastric adenocarcinoma in Asia: a comparison of associations between cardia and non-cardia tumours. Gut. 2016;65:1611-8.

13. Mocellin S, Verdi D, Pooley KA, Nitti D. Genetic variation and gastric cancer risk: a field synopsis and meta-analysis. Gut. 2015;64:1209-19.

14. Taupin D, Podolsky DK. Trefoil factors: initiators of mucosal healing. Nat Rev Mol Cell Biol. 2003;4:721-32.

15. Hoffmann W. Trefoil factors TFF (trefoil factor family) peptide-triggered signals promoting mucosal restitution. Cell Mol Life Sci. 2005;62:2932-8.

16. Peterson AJ, Menheniott TR, O'Connor L, Walduck AK, Fox JG, Kawakami K, Minamoto T, et al. Helicobacter pylori infection promotes methylation and silencing of trefoil factor 2, leading to gastric tumor development in mice and humans. Gastroenterology. 2010;139:2005-17.

17. Ng GZ, Menheniott TR, Every AL, Stent A, Judd LM, Chionh YT, Dhar P, et al. The MUC1 mucin protects against helicobacter pylori pathogenesis in mice by regulation of the NLRP3 inflammasome. Gut. 2016;65:1087-99.

18. Wang G, Hu N, Yang HH, Wang L, Su H, Wang C, Clifford R, et al. Comparison of global gene expression of gastric cardia and noncardia cancers from a high-risk population in China. PLoS One. 2013;8:e63826.

19. Zhu H, Wang Q, Yao Y, Fang J, Sun F, Ni Y, Shen Y, et al. Microarray analysis of long non-coding RNA expression profiles in human gastric cells and tissues with helicobacter pylori infection. BMC Med Genet. 2015;8:84.

20. Gyorffy B, Lanczky A, Eklund AC, Denkert C, Budczies J, Li Q, Szallasi Z. An online survival analysis tool to rapidly assess the effect of 22,277 genes on breast cancer prognosis using microarray data of 1,809 patients. Breast Cancer Res Treat. 2010;123:725-31.

21. Longman RJ, Poulsom R, Corfield AP, Warren BF, Wright NA, Thomas MG. Alterations in the composition of the supramucosal defense barrier in relation to disease severity of ulcerative colitis. J Histochem Cytochem. 2006;54:1335-48.

22. Labouvie C, Machado JC, Carneiro F, Sarbia M, Vieth M, Porschen R, Seitz G, et al. Differential expression of mucins and trefoil peptides in native epithelium, Barrett's metaplasia and squamous cell carcinoma of the oesophagus. J Cancer Res Clin Oncol. 1999;125:71-6.

23. Amieva M, Peek RM Jr. Pathobiology of helicobacter pylori-induced gastric cancer. Gastroenterology. 2016;150:64-78.

24. Naumann M, Sokolova O, Tegtmeyer N, Backert S. Helicobacter pylori: a paradigm pathogen for subverting host cell signal transmission. Trends Microbiol. 2017;25:316-28.

25. Linden SK, Sutton P, Karlsson NG, Korolik V, McGuckin MA. Mucins in the mucosal barrier to infection. Mucosal Immunol. 2008;1:183-97.

26. Vinall LE, King M, Novelli M, Green CA, Daniels G, Hilkens J, Sarner M, et al. Altered expression and allelic association of the hypervariable membrane mucin MUC1 in helicobacter pylori gastritis. Gastroenterology. 2002;123:41-9.
27. Swallow DM, Gendler S, Griffiths B, Corney G, Taylor-Papadimitriou J, Bramwell ME. The human tumour-associated epithelial mucins are coded by an expressed hypervariable gene locus PUM. Nature. 1987;328:82-4.

28. Ligtenberg MJ, Vos HL, Gennissen AM, Hilkens J. Episialin, a carcinomaassociated mucin, is generated by a polymorphic gene encoding splice variants with alternative amino termini. J Biol Chem. 1990;265:5573-8.

29. Saeki N, Saito A, Choi IJ, Matsuo K, Ohnami S, Totsuka H, Chiku S, et al. A functional single nucleotide polymorphism in mucin 1, at chromosome 1q22, determines susceptibility to diffuse-type gastric cancer. Gastroenterology. 2011;140:892-902.

30. Jia Y, Persson C, Hou L, Zheng Z, Yeager M, Lissowska J, Chanock SJ, et al. A comprehensive analysis of common genetic variation in MUC1, MUC5AC, MUC6 genes and risk of stomach cancer. Cancer Causes Control. 2010;21:313-21.

31. Niwa T, Tsukamoto T, Toyoda T, Mori A, Tanaka H, Maekita T, Ichinose M, et al. Inflammatory processes triggered by helicobacter pylori infection cause aberrant DNA methylation in gastric epithelial cells. Cancer Res. 2010; 70:1430-40.

32. Nakamura J, Tanaka T, Kitajima Y, Noshiro H, Miyazaki K. Methylationmediated gene silencing as biomarkers of gastric cancer: a review. World J Gastroenterol. 2014:20:11991-2006.

33. Tan P, Yeoh KG. Genetics and molecular pathogenesis of gastric adenocarcinoma. Gastroenterology. 2015;149:1153-62 e1153.

34. Wright NA, Hoffmann W, Otto WR, Rio MC, Thim L. Rolling in the clover: trefoil factor family (TFF)-domain peptides, cell migration and cancer. FEBS Lett. 1997;408:121-3.

35. Kindon H, Pothoulakis C, Thim L, Lynch-Devaney K, Podolsky DK. Trefoil peptide protection of intestinal epithelial barrier function: cooperative interaction with mucin glycoprotein. Gastroenterology. 1995;109:516-23.

36. Liu J, Wang X, Yang X, Liu Y, Shi Y, Ren J, Guleng B. miRNA423-5p regulates cell proliferation and invasion by targeting trefoil factor 1 in gastric cancer cells. Cancer Lett. 2014;347:98-104.

37. Bossenmeyer-Pourie C, Kannan R, Ribieras S, Wendling C, Stoll I, Thim L, Tomasetto $C$, et al. The trefoil factor 1 participates in gastrointestinal cell differentiation by delaying G1-S phase transition and reducing apoptosis. J Cell Biol. 2002;157:761-70.

38. Fox JG, Rogers AB, Whary MT, Ge Z, Ohtani M, Jones EK, Wang TC. Accelerated progression of gastritis to dysplasia in the pyloric antrum of TFF2 -/- C57BL6 x Sv129 helicobacter pylori-infected mice. Am J Pathol. 2007;171:1520-8.

39. Yamaguchi J, Mino-Kenudson M, Liss AS, Chowdhury S, Wang TC, Fernandez-Del Castillo C, Lillemoe KD, et al. Loss of trefoil factor 2 from pancreatic duct glands promotes formation of intraductal papillary mucinous neoplasms in mice. Gastroenterology. 2016;151:1232-44 e1210.

40. Vestergaard EM, Nexo E, Torring N, Borre M, Orntoft TF, Sorensen KD. Promoter hypomethylation and upregulation of trefoil factors in prostate cancer. Int J Cancer. 2010;127:1857-65.

41. Ishibashi Y, Ohtsu H, Ikemura M, Kikuchi Y, Niwa T, Nishioka K, Uchida Y, et al. Serum TFF1 and TFF3 but not TFF2 are higher in women with breast cancer than in women without breast cancer. Sci Rep. 2017;7:4846.

42. Sasaki M, Ikeda H, Nakanuma Y. Expression profiles of MUC mucins and trefoil factor family (TFF) peptides in the intrahepatic biliary system: physiological distribution and pathological significance. Prog Histochem Cytochem. 2007:42:61-110.

43. Cheng AS, Li MS, Kang W, Cheng VY, Chou JL, Lau SS, Go MY, et al. Helicobacter pylori causes epigenetic dysregulation of FOXD3 to promote gastric carcinogenesis. Gastroenterology. 2013;144:122-33 e129.

44. Cui Y, Gao D, Linghu E, Zhan Q, Chen R, Brock MV, Herman JG, et al. Epigenetic changes and functional study of HOXA11 in human gastric cancer. Epigenomics. 2015;7:201-13.

\section{Publisher's Note}

Springer Nature remains neutral with regard to jurisdictional claims in published maps and institutional affiliations. 